15

\title{
Моделирование источника ультрахолодных нейтронов на реакторе ПИК
}

\author{
() А.К. Фомин, А.П. Серебров \\ Петербургский институт ядерной фризики им. Б.П. Константинова Национального исследовательского центра \\ „Курчатовский институт“, \\ 188300 Гатчина, Ленинградская обл., Россия \\ e-mail: fomin_ak@pnpi.nrcki.ru
}

Поступило в Редакцию 21 сентября 2021 г. В окончательной редакции 9 ноября 2021 г. Принято к публикации 10 ноября 2021 г.

\begin{abstract}
Представлено моделирование комплекса исследований с ультрахолодными нейтронами на реакторе ПИК (Гатчина, Россия). Комплекс создается на базе высокоинтенсивного источника ультрахолодных нейтронов на канале ГЭК-4. Разработана Монте-Карло-модель, включающая в себя источник, нейтроноводную систему и экспериментальную установку по поиску электрического дипольного момента нейтрона с учетом их реального расположения в главном зале реактора. При помощи разработанной компьютерной модели получено значение плотности ультрахолодных нейтронов в установке, которая составила $200 \mathrm{~cm}^{-3}$. Это в 50 раз выше, чем на источнике в Институте Лауэ-Ланжевена (Гренобль, Франция). Такая плотность позволит достичь чувствительности измерений в эксперименте $1 \cdot 10^{-27} \mathrm{e} \cdot \mathrm{cm} /$ year.
\end{abstract}

Ключевые слова: ультрахолодные нейтроны, высокоинтенсивный источник, сверхтекучий гелий.

DOI: $10.21883 /$ JTF.2022.02.52025.261-21

\section{Введение}

В НИЦ „Курчатовский институт“-ПИЯФ создается комплекс исследований с ультрахолодными нейтронами (УХН) на реакторе ПИК [1]. В основе комплекса лежит высокоинтенсивный источник УХН на основе сверхтекучего гелия. Нейтроны очень низких энергий $\left(\sim 10^{-7} \mathrm{eV}\right)$, называемые ультрахолодными, обладают уникальным свойством - их можно хранить в материальных и магнитных ловушках [2-4]. Это дает широкие методические возможности для проведения прецизионных экспериментов и изучения фундаментальных вопросов физики. Условием отражения нейтрона от стенки ловушки являетя $v_{n}<v_{b}$, где $v_{n}$ - проекция вектора скорости на нормаль к поверхности, $v_{b}$ - граничная скорость материала стенки. Также важным свойством УХН является то, что они подвержены воздействию гравитационного поля Земли и двигаются по параболическим траекториям с максимальной высотой подъема ограниченной их энергией. На возможность хранения нейтронов в сосудах было указано Я.Б. Зельдовичем [5], а первый эксперимент по выводу УХН из реактора был осуществлен в 1968 г. в ОИЯИ (Дубна, Россия) [6]. В настоящее время УХН успешно используются для фундаментальных исследований: для поиска электрического дипольного момента (ЭДМ) нейтрона, для измерения времени жизни нейтрона, для измерения асимметрий распада нейтрона и т.д. Точность этих экспериментов ограничена статистикой, поэтому актуальной является задача разработки новых высокоинтенсивных источников УХН [7].
Эксперимент по поиску ЭДМ нейтрона связан с общей проблемой теории элементарных частиц: адекватным описанием процессов, идущих с нарушением СР- и Т-симметрии. Величина ЭДМ в современных теоретических моделях возникает в первом порядке слабого взаимодействия и оказывается на уровне $10^{-26}-10^{-28} \mathrm{e} \cdot \mathrm{cm}$. Поиски ЭДМ нейтрона были инициированы в 1950-х годах [8]. С дальнейшей историей измерений можно ознакомиться в Particle Data Group [9]. Лучшее экспериментальное ограничение на величину ЭДМ нейтрона на сегодняшний день составляет $|d n|<1.8 \cdot 10^{-26} \mathrm{e} \cdot \mathrm{cm}$ (90\% C.L.) [10]. На реакторе ПИК измерения будут проводиться при помощи дифференциального магниторезонансного спектрометра с двумя камерами хранения УХН [11]. Для получения абсолютного значения плотности УХН в ЭДМ спектрометре разработана МонтеКарло модель эксперимента на реакторе ПИК.

\section{1. Источник УХН на основе сверхтекучего гелия}

Основной элемент источника - камера, заполненная сверхтекучим гелием при температуре $1 \mathrm{~K}$, который является конвертером холодных нейтронов в УХН. Источник располагается на пучке канала ГЭК-4. Ультрахолодные нейтроны рождаются в гелии из холодных нейтронов с длиной волны $9 \AA$. При этом нейтроны, скорость которых превосходит граничную скорость покрытия стенок камеры, проходят через стенку, а УХН накапливаются до плотности, определяемой временем хранения в камере, и могут быть выведены по нейтро- 


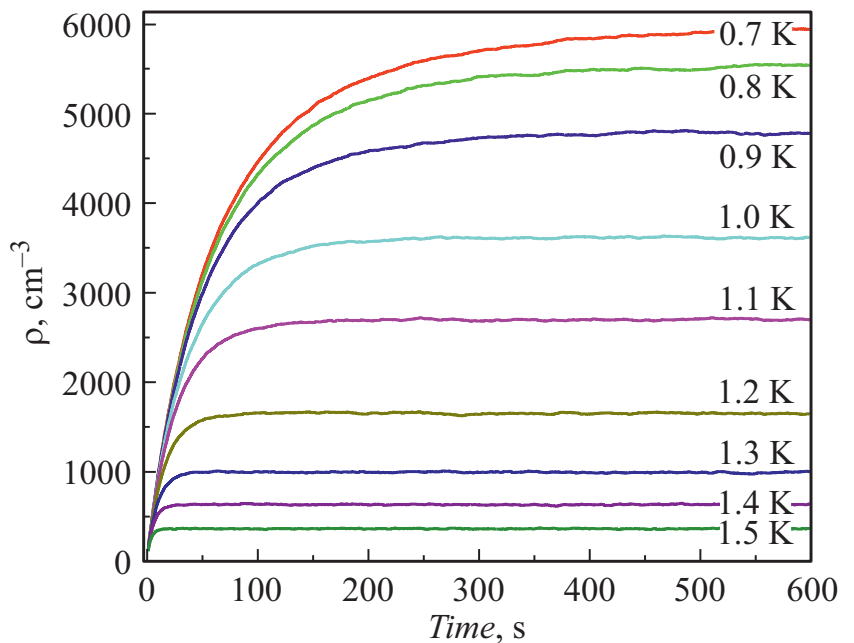

Рис. 1. Динамика накопления УХН в закрытой камере источника при разной температуре сверхтекучего гелия. Коэффициент потерь для напыления стенки камеры равен $3 \cdot 10^{-4}$.

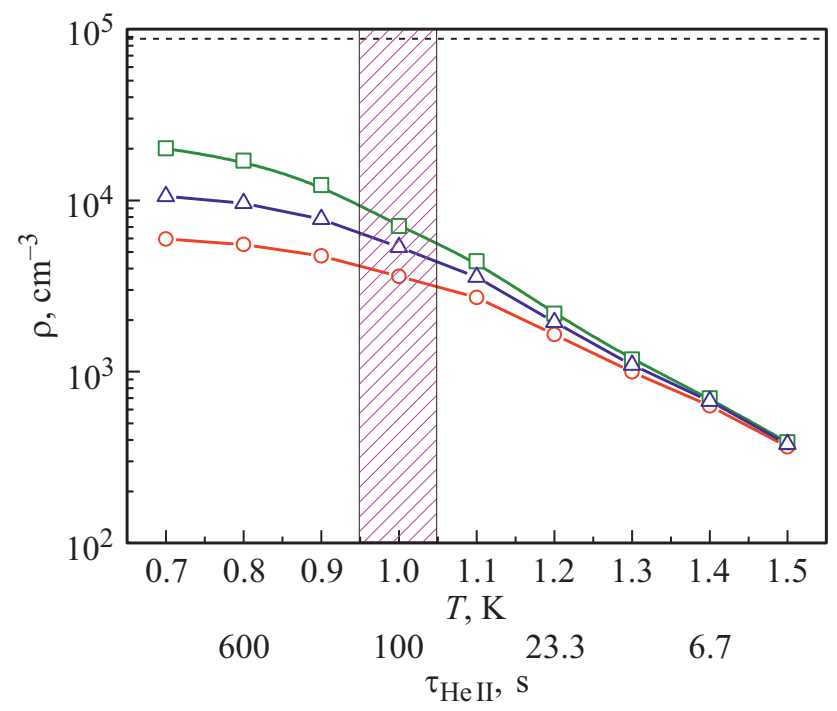

Рис. 2. Плотность УХН в закрытой камере источника в зависимости от температуры сверхтекучего гелия в камере источника. Коэффициент потерь для напыления стенки камеры: квадраты -0 , треугольники $-1 \cdot 10^{-4}$, круги $-3 \cdot 10^{-4}$. Штриховая линия - плотность УХН, которая может быть достигнута при отсутствии потерь при соударениях со стенками и в сверхтекучем гелии.

новоду в экспериментальную установку. Время хранения определяется температурой сверхтекучего гелия и коэффициентом потерь при соударениях нейтронов со стенками. Время хранения нейтронов в сверхтекучем гелии растет с уменьшением температуры и составляет $100 \mathrm{~s}$ при температуре $1 \mathrm{~K}$ [12].

Далее представлены результаты расчетов достижимой плотности УХН в закрытом объеме камеры. Камера имеет внутри напыление ${ }^{58} \mathrm{NiMo} \mathrm{c} \mathrm{граничной} \mathrm{скоро-}$ стью $7.8 \mathrm{~m} / \mathrm{s}$ и коэффициентом потерь $3 \cdot 10^{-4}$. При
$\Phi(\lambda=9 \AA)=1 \cdot 10^{9} \mathrm{~cm}^{-2} \mathrm{~s}^{-1} \AA^{-1}, \quad$ скорость производства УХН в сверхтекучем гелии составляет $100 \mathrm{~cm}^{-3} \mathrm{~s}^{-1}$. Объем камеры источника составляет 351. Диаметр трубопровода подачи жидкого гелия $1 \mathrm{~cm}$. Отвод теплопритоков от сверхтекучего гелия осуществляется через теплообменник. Динамика накопления УХН в камере источника при разных температурах показана на рис. 1. Статистические неопределенности, полученные в расчетах, составляют менее $0.5 \%$ и в связи с этим не показаны на приведенных рисунках. Плотность УХН в установившемся режиме в зависимости от температуры для разных значений коэффициента потерь показана на рис. 2. Плотность УХН в закрытой камере источника при температуре $1 \mathrm{~K}$ и коэффициенте потерь для напыления стенок камеры $3 \cdot 10^{-4}$ составляет $3.5 \cdot 10^{3} \mathrm{~cm}^{-3}$.

\section{2. Испытательный стенд}

Для изготовления источника УХН важно обеспечить соответствующее качество его составных элементов. Плотность УХН в эксперименте будет тем выше, чем лучше будет хранение нейтронов в камере источника и чем лучше будет пропускание нейтроноводной системы. Для тестирования элементов конструкции источника разработан специальный испытательный стенд (рис. 3), который может использоваться на существующих источниках УХН. Стенд можно использовать для тестирования хранения УХН в камере источника (рис. 3, $a$ ), либо для тестирования пропускания через нейтроноводную систему от источника к экспериментальной установке (рис. $3, b)$. При этом нейтроноводная система может включать такие элементы, как повороты, разветвительные тройники, мембранные узлы и т.д. Разработана
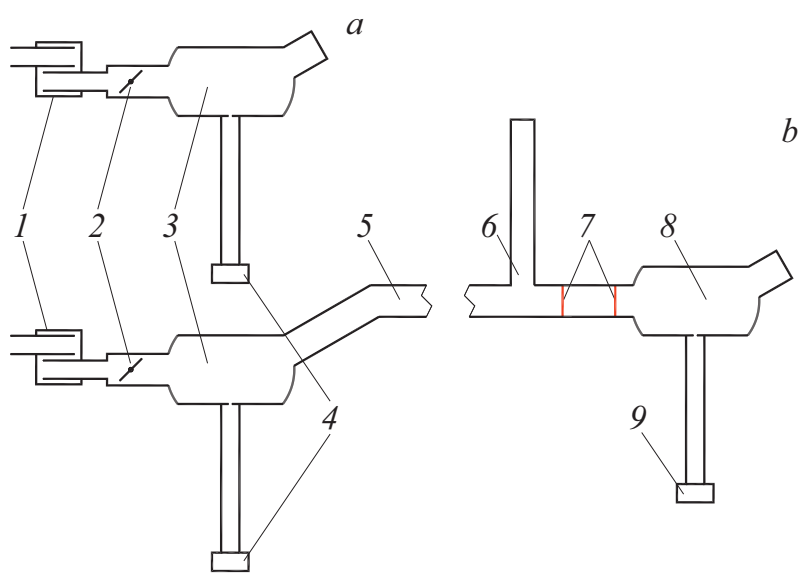

Рис. 3. Схемы сборки испытательного стенда: $a-$ для тестирования хранения УХН в камере, $b$ - для тестирования пропускания УХН через нейтроноводную систему. 1 - турбулизатор, 2 - затвор, 3 - камера хранения № 1 , 4 - детектор УХН камеры хранения № $1,5-$ нейтроновод, 6 - тройник, 7 - Al-мембраны, 8 - камера хранения № 2, 9 - детектор УХН камеры хранения № 2 . 
Монте-Карло-модель стенда, позволяющая произвести расчеты при различных режимах его работы.

На входе в стенд установлен турбулизатор 1, предназначенный для обрезки надбарьерных нейтронов в спектре [13]. Турбулизатор представляет собой камеру, в которую входят с разных сторон два нейтроновода так, чтобы исключить прямой пролет нейтронов из одного нейтроновода в другой. Таким образом, нейтроны, проходя через него, совершают несколько соударений, и надбарьерные нейтроны с большой степенью вероятности отсеиваются. Турбулизатор изготовлен из нержавеющей стали с граничной скоростью $6.2 \mathrm{~m} / \mathrm{s}$ и коэффициентом потерь $3 \cdot 10^{-4}$. На входе в турбулизатор разыгрывался спектр Максвелла. После турбулизатора нейтроны попадают в нейтроновод с затвором 2. Диаметр нейтроновода составляет $138 \mathrm{~mm}$, диаметр затвора - $137 \mathrm{~mm}$. После нейтроновода нейтроны попадают в камеру хранения № 1 3. Камера и нейтроноводы напылены NiMo c граничной скоростью $6.55 \mathrm{~m} / \mathrm{s}$ и коэффициентом потерь $3 \cdot 10^{-4}[14]$. Снизу к камере подсоединен нейтроновод, ведущий к детектору УХН камеры хранения № 14 . Диаметр выходного отверстия из камеры составляет $11 \mathrm{~mm}$. Далее к камере подсоединен либо заглушенный переходник (рис. $3, a$ ), либо нейтроновод 5 диаметром $138 \mathrm{~mm}$ (рис. 3, b). В нейтроноводе установлены тройник 6 и две Al мембраны 7. К другому концу нейтроновода подсоединена камера хранения № 28 с детектором УХН 9, которые по конструкции аналогичны камере 3 с детектором 4. Отражения УХН от стенок нейтроноводов являются в основном зеркальными, вероятность диффузного отражения составляет $0.7 \%$. При отражении УХН от стенок камер вероятность диффузного отражения составляет $90 \%$.

\section{1. Моделирование хранения нейтронов в камере источника}

При моделировании хранения нейтронов в камере источника конструкция подсоединения элементов стенда соответствует рис. 3, a. Соответствующий счет детектора УХН 4 представлен на рис. 4. Нейтроны начинают поступать от источника нейтронов в постоянном режиме в момент времени $t=0 \mathrm{~s}$. При этом появляется небольшой счет детектора, так как нейтроны попадают в камеру 3 через зазор в затворе 2. Затвор открыт в интервале времени от 200 до $300 \mathrm{~s}$. При открытии затвора происходит увеличение счета детектора 4. Расчет произведен для нескольких значений коэффициента потерь покрытия камеры источника. Таким образом, по характеру счета детектора можно сделать вывод о качестве покрытия.

\section{2. Моделирование пропускания через нейтроноводную систему}

При моделировании пропускания через нейтроноводную систему конструкция подсоединения элементов

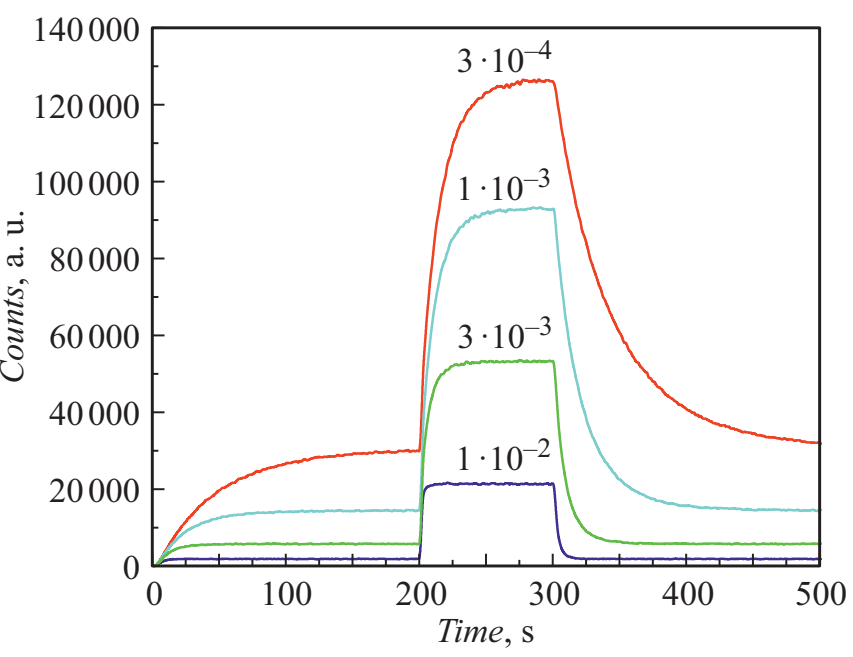

Рис. 4. Счет детектора при моделировании хранения в камере для разных значений коэффициента потерь покрытия стенок.

стенда соответствует рис. $3, b$. Нейтроновод 5 выходит из камеры 3 под углом $30^{\circ}$, а затем поворачивает в горизонтальное положение. В источнике УХН такая конструкция сделана для сохранения уровня сверхтекучего гелия в камере. Суммарная длина нейтроновода составляет $3.5 \mathrm{~m}$. Перед камерой № 2 установлены тройник 6 и две Al-мембраны 7. Включение тройника в систему обусловлено тем, что одной из возможных конструкций источника является вариант с охлаждением сверхтекучего гелия путем откачки его паров через трубопровод. Трубопровод откачки в этом случае подходит к нейтроноводу вертикально и одновременно представляет собой гравитационный затвор для УХН. Al-мембраны предназначены для разделения объемов по вакууму. Так как на источнике планируется установить несколько экспериментальных установок, то УХН будут перенаправляться из нейтроновода источника к установкам через разветвитель пучка. Соответственно первая мембрана относится к источнику УХН, а вторая - к экспериментальной установке. Толщина мембран составляет $100 \mu \mathrm{m}$, граничная скорость $3.2 \mathrm{~m} / \mathrm{s}$. Коэффициент потерь для напыления стенок камер хранения в данном расчете составляет $3 \cdot 10^{-4}$. Соответствующие счета детекторов УХН 4 и 9 представлены на рис. 5 для разного количества мембран. Затвор открыт в интервале времени от 200 до $400 \mathrm{~s}$. Оценка пропускания нейтроноводной системы с различными элементами делается исходя из разницы счетов детекторов УХН 4 и 9. Таким образом, для заданной конфигурации пропускание составляет $\sim 75 \%$ при отсутствии мембран, $\sim 53 \%-$ с одной мембраной, $38 \%$ - с двумя мембранами.

\section{3. Плотность УХН в ЭДМ спектрометре}

Для поиска ЭДМ нейтрона будет использован дифференциальный магниторезонансный спектрометр с дву- 


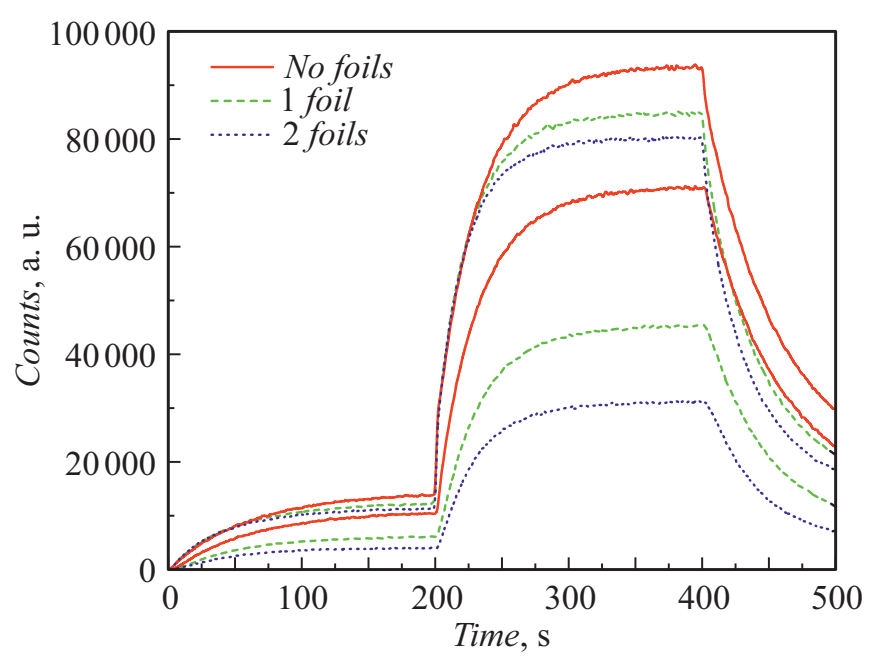

Рис. 5. Временная диаграмма счетов детекторов для вариантов нейтроноводной системы: без мембран, с одной мембраной и с двумя мембранами. Три верхние кривые соответствуют счету детектора камеры № 1, три нижние - счету детектора камеры № 2.

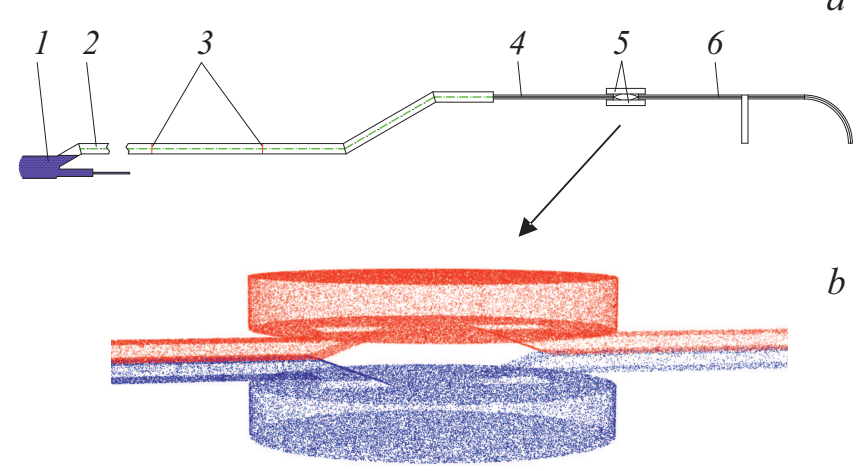

Рис. 6. $a-$ расчетная схема эксперимента по поиску ЭДМ нейтрона на реакторе ПИК: 1 - камера источника УХН с сверхтекучим гелием, 2 - нейтроновод диаметром $140 \mathrm{~mm}, 3$ - две Al-фольги, 4 - два нейтроновода сечением $25 \times 85 \mathrm{~mm}, 5$ - камеры ЭДМ спектрометра, $6-$ нейтроноводы, ведущие к четырем детекторам. $b$ - точки соударения нейтронов со стенками камер ЭДМ спектрометра, полученные в результате работы Монте-Карло-модели.

мя камерами хранения УХН, реверсируемым электрическим полем, системой двойного анализа поляризации и четырьмя детекторами [11]. Статистическая точность измерений в экспреименте определяется формулой $\delta d_{n}=\frac{h}{4 \pi \alpha E T \sqrt{N}}$, где $\alpha$ - параметр качества резонансной кривой, $E-$ напряженность электрического поля, $T-$ время хранения нейтронов в резонансных условиях, $N$ - полный счет нейтронов за время эксперимента. Для оценки чувствительности экперимента на реакторе ПИК необходимо получить расчетное значение плотности УХН в установке.
Расчетная схема эксперимента на реакторе ПИК представлена на рис. 6, $a$. От источника идет прямой нейтроновод диаметром $140 \mathrm{~mm}$, который затем переходит в два нейтроновода сечением $25 \times 85 \mathrm{~mm}$, ведущих к камерам ЭДМ спектрометра. Расстояние от камеры источника до камер установки составляет около $13 \mathrm{~m}$. Вход нейтроноводов в спектрометр, а также их выход происходит через центральный электрод. Камера источника и нейтроноводы имеют внутри напыление ${ }^{58} \mathrm{NiMo} \mathrm{c} \mathrm{граничной} \mathrm{скоростью} 7.8 \mathrm{~m} / \mathrm{s}$ и коэффициентом потерь $3 \cdot 10^{-4}$. Ловушки спектрометра имеют радиус $263 \mathrm{~mm}$ и высоту $76 \mathrm{~mm}$. Граничная скорость покрытия из бериллия составляет $6.8 \mathrm{~m} / \mathrm{s}$, коэффициент потерь $1.2 \cdot 10^{-4}$. Разработана Монте-Карло-модель эксперимента, в которой разыгрывается история каждого нейтрона от момента рождения в источнике до попадания в экспериментальный объем с учетом всех возможных потерь. Для иллюстрации работы Монте-Карло-модели на рис. $6, b$ показаны точки соударения нейтронов со стенками камер ЭДМ спектрометра, полученные в результате расчетов.

Для восстановления части спектра, обрезанного снизу Al-фольгой, рассмотрены варианты с поднятием ЭДМ спектрометра путем установки его на платформу. При этом мы получаем более мягкий спектр УХН, который имеет лучшее время хранения. При поднятии угол поворота нейтроновода составляет $30^{\circ}$. Рассчитаны варианты с поднятием на $35,70,105 \mathrm{~cm}$. На рис. 7 показана динамика изменения плотности УХН в ЭДМ спектрометре с течением времени. При поднятии выше $35 \mathrm{~cm}$ начинает сказываться эффект обрезки спектра сверху в камере источника УХН, что приводит к уменьшению плотности УХН в ЭДМ спектрометре. Соответствующие нейтронные спектры показаны на рис. 8. В результате моделирования получено, что в эксперименте на реакторе ПИК может быть достигнуто значение плотности $\sim 200 \mathrm{~cm}^{-3}$.

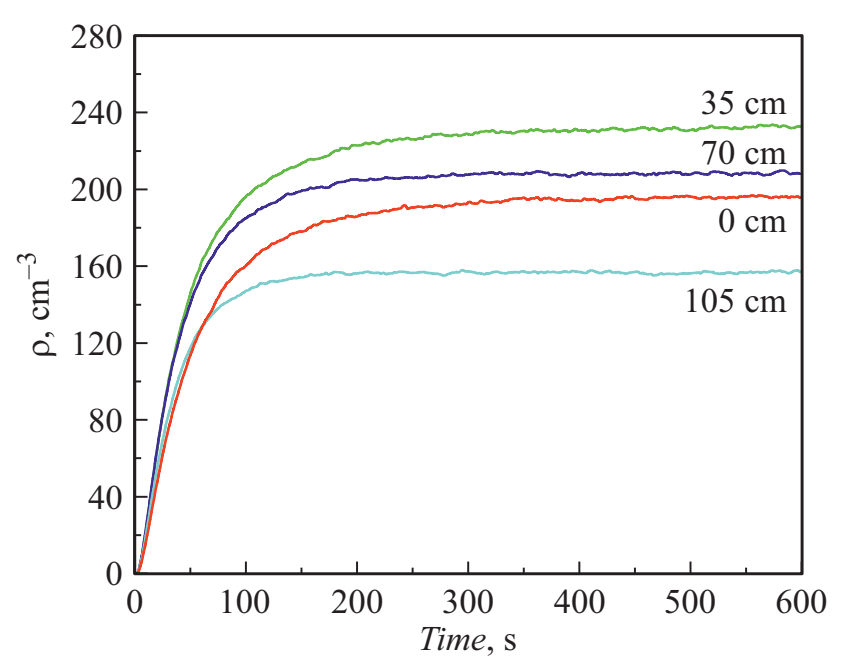

Рис. 7. Динамика изменения плотности УХН в ЭДМ спектрометре при разной высоте поднятия установки. 


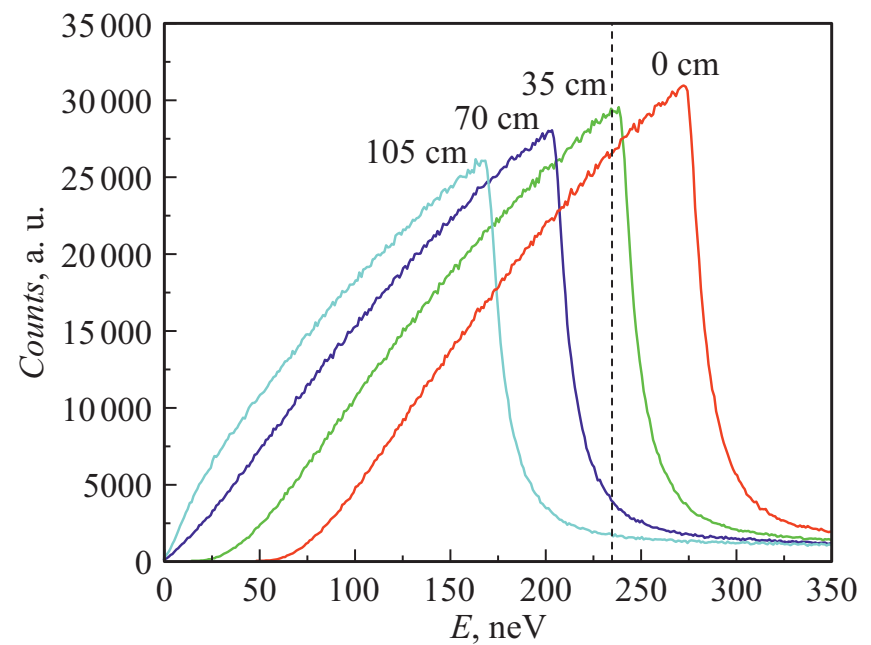

Рис. 8. Спектры нейтронов в нейтроноводе перед ЭДМ спектрометром при разной высоте поднятия установки. Штриховая линия - граничная энергия покрытия камер спектрометра, составляющая $235 \mathrm{neV}$.

\section{Заключение}

При помощи разработанной компьютерной модели получено значение плотности УХН в ЭДМ спектрометре, которая составила $200 \mathrm{~cm}^{-3}$. На реакторе в Институте Лауэ-Ланжевена (ИЛЛ) (Гренобль, Франция) на ЭДМ спектрометре была получена чувствительность $\delta d_{n} \sim 1.7 \cdot 10^{-25} \mathrm{e} \cdot \mathrm{cm} /$ day при плотности УХН на входе в спектрометр $4 \mathrm{~cm}^{-3}$. При увеличении напряженности электрического поля с $12-14$ до $27 \mathrm{kV} / \mathrm{cm}$ [15] чувствительность эксперимента улучшается до $\delta d_{n} \sim 1 \cdot 10^{-25} \mathrm{e} \cdot \mathrm{cm} /$ day на реакторе ИЛЛ. При плотности УХН в камерах спектрометра $200 \mathrm{~cm}^{-3}$ на реакторе ПИК может быть получена чувствительность $\delta d_{n} \sim 1 \cdot 10^{-27} \mathrm{e} \cdot \mathrm{cm} /$ year. Обнаружение ЭДМ нейтрона либо новое ограничение на его величину на таком уровне может стать решающим фактором при выборе теории, адекватно описывающей явления нарушения СР-симметрии.

\section{Финансирование работы}

Работа выполнена в рамках работ по созданию приборной базы реактора ПИК.

\section{Конфликт интересов}

Авторы заявляют, что у них нет конфликта интересов.

\section{Список литературы}

[1] M.V. Kovalchuk, V.V. Voronin, S.V. Grigoriev, A.P. Serebrov. Crystallogr. Rep., 66 (2), 195 (2021).

DOI: $10.1134 / \mathrm{S} 1063774521020061$
[2] V. Ignatovich. The Physics of Ultracold Neutrons (Clarendon, Oxford, 1990)

[3] R. Golub, D. Richardson, S. Lamoreaux. Ultra-Cold Neutrons (Adam Hilger, Bristol, Philadelphia, NY., 1991)

[4] A. Steyerl. Ultracold Neutrons (World Scientific Publishing Company, Singapore, 2020)

[5] Я.Б. Зельдович. ЖЭТФ, 36, 1952 (1959).

[6] В.И. Лущиков, Ю.Н. Покотиловский, А.В. Стрелков, Ф.Л. Шапиро. Письма в ЖЭТФ, 9, 40 (1969).

[7] G. Bison, M. Daum, K. Kirch, B. Lauss, D. Ries, P. SchmidtWellenburg, G. Zsigmond, T. Brenner, P. Geltenbort, T. Jenke, O. Zimmer, M. Beck, W. Heil, J. Kahlenberg, J. Karch, K. Ross, K. Eberhardt, C. Geppert, S. Karpuk, T. Reich, C. Siemensen, Y. Sobolev, N. Trautmann. Phys. Rev. C, 95, 045503 (2017). DOI: 10.1103/PhysRevC.95.045503

[8] J.H. Smith, E.M. Purcell, N.F. Ramsey. Phys. Rev., 108, 120 (1957). DOI: 10.1103/PhysRev.108.120

[9] P.A. Zyla, R.M. Barnett, J. Beringer, O. Dahl, D.A. Dwyer, D.E. Groom, C.J. Lin, K.S. Lugovsky, E. Pianori, D.J. Robinson, C.G. Wohl, W.-M. Yao, K. Agashe, G. Aielli, B.C. Allanach, C. Amsler, M. Antonelli, E.C. Aschenauer, D.M. Asner, H. Baer, Sw. Banerjee, L. Baudis, C.W. Bauer, J.J. Beatty, V.I. Belousov, S. Bethke, A. Bettini, O. Biebel, K.M. Black, E. Blucher, O. Buchmuller, V. Burkert, M.A. Bychkov, R.N. Cahn, M. Carena, A. Ceccucci, A. Cerri, D. Chakraborty, R. Sekhar Chivukula, G. Cowan, G. D’Ambrosio, T. Damour, D. de Florian, A. de Gouvêa, T. DeGrand, P. de Jong, G. Dissertori, B.A. Dobrescu, M. D’Onofrio, M. Doser, M. Drees, H.K. Dreiner, P. Eerola, U. Egede, S. Eidelman, J. Ellis, J. Erler, V.V. Ezhela, W. Fetscher, B.D. Fields, B. Foster, A. Freitas, H. Gallagher, L. Garren, H.-J. Gerber, G. Gerbier, T. Gershon, Y. Gershtein, T. Gherghetta, A.A. Godizov, M.C. Gonzalez-Garcia, M. Goodman, C. Grab, A.V. Gritsan, C. Grojean, M. Grünewald, A. Gurtu, T. Gutsche, H.E. Haber, C. Hanhart, S. Hashimoto, Y. Hayato, A. Hebecker, S. Heinemeyer, B. Heltsley, J.J. HernándezRey, K. Hikasa, J. Hisano, A. Höcker, J. Holder, A. Holtkamp, J. Huston, T. Hyodo, K.F. Johnson, M. Kado, M. Karliner, U.F. Katz, M. Kenzie, V.A. Khoze, S.R. Klein, E. Klempt, R.V. Kowalewski, F. Krauss, M. Kreps, B. Krusche, Y. Kwon, O. Lahav, J. Laiho, L.P. Lellouch, J. Lesgourgues, A.R. Liddle, Z. Ligeti, C. Lippmann, T.M. Liss, L. Littenberg, C. Lourengo, S.B. Lugovsky, A. Lusiani, Y. Makida, F. Maltoni, T. Mannel, A.V. Manohar, W.J. Marciano, A. Masoni, J. Matthews, U.-G. Meißner, M. Mikhasenko, D.J. Miller, D. Milstead, R.E. Mitchell, K. Mönig, P. Molaro, F. Moortgat, M. Moskovic, K. Nakamura, M. Narain, P. Nason, S. Navas, M. Neubert, P. Nevski, Y. Nir, K.A. Olive, C. Patrignani, J.A. Peacock, S.T. Petcov, V.A. Petrov, A. Pich, A. Piepke, P.A. Zyla, R.M. Barnett, J. Beringer, O. Dahl, D.A. Dwyer, D.E. Groom, C.-J. Lin, K.S. Lugovsky, E. Pianor A. Pomarol, S. Profumo, A. Quadt, K. Rabbertz, J. Rademacker, G. Raffelt, H. Ramani, M. Ramsey-Musolf, B.N. Ratcliff, P. Richardson, A. Ringwald, S. Roesler, S. Rolli, A. Romaniouk, L.J. Rosenberg, J.L. Rosner, G. Rybka, M. Ryskin, R.A. Ryutin, Y. Sakai, G.P. Salam, S. Sarkar, F. Sauli, O. Schneider, K. Scholberg, A.J. Schwartz, J. Schwiening, D. Scott, V. Sharma, S.R. Sharpe, T. Shutt, M. Silari, T. Sjöstrand, P. Skands, T. Skwarnicki, G.F. Smoot, A. Soffer, M.S. Sozzi, S. Spanier, C. Spiering, 


\begin{abstract}
A. Stahl, S.L. Stone, Y. Sumino, T. Sumiyoshi, M.J. Syphers, F. Takahashi, M. Tanabashi, J. Tanaka, M. Taševský, K. Terashi, J. Terning, U. Thoma, R.S. Thorne, L. Tiator, M. Titov, N.P. Tkachenko, D.R. Tovey, K. Trabelsi, P. Urquijo, G. Valencia, R. Van de Water, N. Varelas, G. Venanzoni, L. Verde, M.G. Vincter, P. Vogel, W. Vogelsang, A. Vogt, V. Vorobyev, S.P. Wakely, W. Walkowiak, C.W. Walter, D. Wands, M.O. Wascko, D.H. Weinberg, E.J. Weinberg, M. White, L.R. Wiencke, S. Willocq, C.L. Woody, R.L. Workman, M. Yokoyama, R. Yoshida, G. Zanderighi, G.P. Zeller, O.V. Zenin, R.-Y. Zhu, S.-L. Zhu, F. Zimmermann, J. Anderson, T. Basaglia, V.S. Lugovsky, P. Schaffner, W. Zheng. Prog. Theor. Exp. Phys., 2020, 083C01 (2020-2021).

DOI: $10.1093 /$ ptep/ptaa104
\end{abstract}

[10] C. Abel, S. Afach, N.J. Ayres, C.A. Baker, G. Ban, G. Bison, K. Bodek, V. Bondar, M. Burghoff, E. Chanel, Z. Chowdhuri, P.-J. Chiu, B. Clement, C.B. Crawford, M. Daum, S. Emmenegger, L. Ferraris-Bouchez, M. Fertl, P. Flaux, B. Franke, A. Fratangelo, P. Geltenbort, K. Green, W.C. Griffith, M. van der Grinten, Z.D. Grujić, P.G. Harris, L. Hayen, W. Heil, R. Henneck, V. Hélaine, N. Hild, Z. Hodge, M. Horras, P. Iaydjiev, S.N. Ivanov, M. Kasprzak, Y. Kermaidic, K. Kirch, A. Knecht, P. Knowles, H.-C. Koch, P.A. Koss, S. Komposch, A. Kozela, A. Kraft, J. Krempel, M. Kuḱniak, B. Lauss, T. Lefort, Y. Lemiére, A. Leredde, P. Mohanmurthy, A. Mtchedlishvili, M. Musgrave, O. NaviliatCuncic, D. Pais, F.M. Piegsa, E. Pierre, G. Pignol, C. Plonka-Spehr, P.N. Prashanth, G. Quéméner, M. Rawlik, D. Rebreyend, I. Rienäcker, D. Ries, S. Roccia, G. Rogel, D. Rozpedzik, A. Schnabel, P. Schmidt-Wellenburg, N. Severijns, D. Shiers,. Tavakoli Dinani, J.A. Thorne, R. Virot, J. Voigt, A. Weis, E. Wursten, G. Wyszynski, J. Zejma, J. Zenner, G. Zsigmond. Phys. Rev. Lett., 124, 081803 (2020). DOI: 10.1103/PhysRevLett.124.081803

[11] A.P. Serebrov, E.A. Kolomenskiy, A.N. Pirozhkov, I.A. Krasnoschekova, A.V. Vassiljev, A.O. Polyushkin, M.S. Lasakov, A.N. Murashkin, V.A. Solovey, A.K. Fomin, I.V. Shoka, O.M. Zherebtsov, P. Geltenbort, S.N. Ivanov, O. Zimmer, E.B. Alexandrov, S.P. Dmitriev, N.A. Dovator. Phys. Rev. C, 92, 055501 (2015). DOI: 10.1103/PhysRevC.92.055501

[12] R. Golub, C. Jewell, P. Ageron, W. Mampe, B. Heckel, I. Kilvington. Z. Phys. B Con. Mat., 51, 187 (1983). DOI:10.1007/BF01307673

[13] M. Daum, A. Frei, P. Geltenbort, E. Gutsmiedl, P. Höbel, H.-C. Koch, A. Kraft, T. Lauer, A.R. Müller, S. Paul, G. Zsigmond. Nucl. Instrum. Meth. A, 675, 103 (2012). DOI: 10.1016/j.nima.2012.02.007

[14] V. Bondar, S. Chesnevskaya, M. Daum, B. Franke, P. Geltenbort, L. Göltl, E. Gutsmiedl, J. Karch, M. Kasprzak, G. Kessler, K. Kirch, H.-C. Koch, A. Kraft, T. Lauer, B. Lauss, E. Pierre, G. Pignol, D. Reggiani, P. Schmidt-Wellenburg, Yu. Sobolev, T. Zechlau, G. Zsigmond. Phys. Rev. C, 96, 035205 (2017). DOI: 10.1103/PhysRevC.96.035205

[15] М.С. Ласаков, А.Н. Пирожков, А.П. Серебров. ЖТФ, 89 (3), 475 (2019) DOI: 10.21883/JTF.2022.02.52025.261-21

[M.S. Lasakov, A.N. Pirozhkov, A.P. Serebrov. Tech. Phys., 64 (3), 436 (2019). DOI: 10.1134/S1063784219030162] 